\title{
Mixed Signal Detection and Carrier Frequency Estimation based on Spectral Coherent Features
}

\author{
Dong $\mathrm{Li}^{1}$, Lin Zhang ${ }^{2}$, Zhiqiang $\mathrm{Liu}^{3}$, Zhiqiang $\mathrm{Wu}^{1}$ and Zhiping Zhang ${ }^{1}$ \\ Department of Electrical Engineering, Wright State University ${ }^{1}$ \\ School of Information Science and Technology, Sun Yat-sen University ${ }^{2}$ \\ Acoustic Division, Code 7160, Naval Research Laboratory ${ }^{3}$ \\ Email: Zhang.176@wright.edu
}

\begin{abstract}
Signal detection and RF parameter estimation have received strong interest in recent years due to the need of spectrum sensing in rapidly growing cognitive radio network research. In most of existing work, the target signal is often assumed to be a single primary user signal without overlap in spectrum with other signals. However, in a spectrally congested environment such as cognitive radio network, or in a spectrally contested environment such as a battlefield, multiple signals are often mixed together with significant overlap in spectrum. In our previous work, we have demonstrated the feasibility of using second order spectrum correlation function (SCF) cyclostationary feature to perform mixed signal detection. In this paper, we extend our work to employ a robust algorithm to detect mixed signals and estimate their carrier frequencies via spectral coherence function (SOF) features. We also evaluate the detection and estimation performances of the proposed algorithm in various channel conditions and signal mixture scenarios. Simulation results confirm the effectiveness of the proposed scheme.
\end{abstract}

\section{INTRODUCTION}

With the rapid growing of cognitive radio network research, spectrum sensing has been investigated thoroughly in the past decade to detect the existence of primary users and spectrum holes to allow secondary user transmission. To allow a better co-existence of primary users and secondary users, it has been recognized that more signal characteristics are needed in addition to the mere existence of the target signals [1] [2]. More sophisticated signal detection/radio frequency (RF) parameter estimation/modulation identification algorithms have been proposed to not only detect the existence of the target signal but obtain important characteristics of the target signal [3]- [9]. However, in most of existing work, the target signal is often assumed to be a single signal without overlap in spectrum with other signals. This assumption is becoming more and more invalid with the recent development of cognitive radio network since multiple secondary users might try to grasp the same spectrum hole and such signals also need to be detected and analyzed to avoid interference. Hence, it is highly desired to develop effective scheme to detect the existence of multiple users signals, identify how many signals are mixed together, and estimate their individual RF parameters.

In our previous work [10], we have demonstrated the feasibility of using second order spectrum correlation function (SCF) cyclostationary feature [11]- [15] to perform mixed signal detection and RF parameter estimation. Here, we extend this work to employ a robust algorithm to detect mixed signals and estimate their carrier frequencies via spectral coherence function (SOF) features [11]- [15]. By employing SOF features, we are able to find a stable threshold in our mixed signal detection algorithm to determine how many mixed components are present. We have evaluated the detection and carrier frequency estimation performances under various channel conditions and signal mixture scenarios. Simulation results confirm the effectiveness and robustness of our algorithm.

The rest of the paper is organized as follows: Section II describes the system model of the mixed signal detection. Section III briefly reviews the second-order cyclostationary feature and discusses the mixed signal detection algorithm based on spectral coherence function (SOF). In Section IV, we evaluate the detection and carrier frequency estimation performances of the proposed algorithm and demonstrate the effectiveness and robustness of the algorithm in various channel conditions and signal mixture scenarios. Simulation results at different signal to noise ratio and channel conditions validate the advantage and reliability of the proposed method. Conclusion follows.

\section{MiXed Signal Detection}

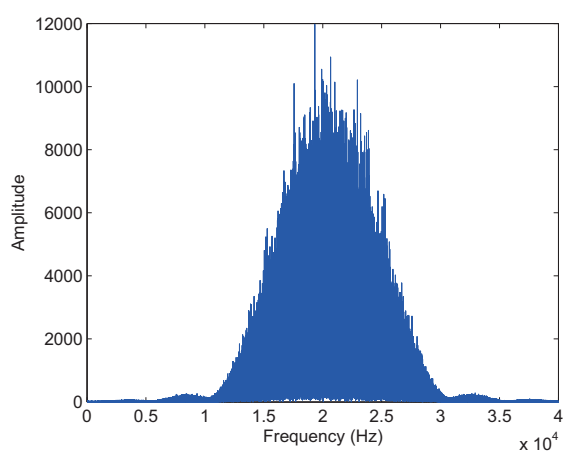

Fig. 1. Spectrum of two different BPSK signals with $95 \%$ spectrum overlap

In a spectrally congested or spectrally contested environment, multiple signals may overlap significantly in frequency domain. Fig. 1 shows the spectrum of a mixed signal with two narrowband BPSK signal components with very close 
carrier frequencies so that there is a 95\% spectrum overlap (the spectrum overlap is defined as the overlapping spectrum divided by the bandwidth of the individual signal). It is obvious that we can no longer tell that there are two signal components by simple frequency analysis, and we cannot use a narrowband filter to distinguish one signal from the other.

\section{SOF based MiXed Signal Detection And CARRIER FREQUENCY ESTIMATION}

\section{A. Second-order Cyclostationary Features and Spectral Co- herence Function}

It has been recognized that many man-made signals, such as communication signal and radar signal, are cyclostationary random processes. The first-order or second-order statistic characteristics (average value, correlation function, etc) of such man-made signals often show periodicity. The periodic features of these signals are not reflected in the conventional power spectrum. By exploiting the correlation characteristics between different frequency bands, we can reveal these periodic features and perform signal detection, RF parameter estimation, and even modulation detection [3]- [9].

In our previous work [10], we have demonstrated the feasibility of detecting mixed signals with significant spectrum overlap using second order cyclostationary spectrum correlation function (SCF) features. However, in [10], the SCF based algorithm doesn't provide a fixed threshold value for detection and a heuristic algorithm needs to be performed to find appropriate detection threshold.

Here, we use cyclostationary spectrum coherence function (SOF) which is a normalized version of SCF to solve this problem. Assume $x(t)$ is a cyclostationary signal, its SOF is defined as [11]- [15]:

$$
C_{X}^{\alpha}(f)=\frac{S_{X}^{\alpha}(f)}{\left[S_{X}^{0}\left(f+\frac{1}{2} \alpha\right)^{*} S_{X}^{0}\left(f-\frac{1}{2} \alpha\right)\right]^{1 / 2}}
$$

where $\alpha$ is the cyclic frequency, $f$ is the spectrum frequency. $S_{x}^{\alpha}(f)$ is the SCF, which is the Fourier transformation of cyclic autocorrelation function $R_{x}^{\alpha}(\tau)$ :

$$
S_{x}^{\alpha}(f)=\int_{-\infty}^{\infty} R_{x}^{\alpha}(\tau) e^{-j 2 \pi f \tau} d \tau .
$$

It is evident that the SOF is upper-bounded by unity and can help to remove channel effect [12]:

$$
\left|C_{X}^{\alpha}(f)\right| \leqslant 1 .
$$

Additionally, SOF has an invariance property [12], which means it will not be affected by linear time-invariant transformations. Hence, we can use SOF and find a universal threshold for our mixed signal detection purpose.

\section{B. Mixed Signal Detection based on SOF}

Similar to SCF, SOF is a two variable (frequency $f$ and cyclic frequency $\alpha$ ) function. Here, we just focus on $f=0$ projection of the SOF because for typical communication signals, the SOF exhibits special features in the cyclic frequency domain when $f=0$. Assume the received mixed signal is composed by two independent BPSK signals with carrier frequencies $f_{c_{1}}$ and $f_{c_{2}}$, which are different but very close to each other. Also, we assume that they have different but close symbol rates. The two signals have independent amplitudes, phases and initial time delays, and they transmit through two independent fading channels.

After normalization of $S_{a}^{\alpha}(f)$ using Equation 1, we can obtain the SOF $C_{a}^{\alpha}(f)$. Next, we use the following three-step algorithm to detect signals and estimate carrier frequencies.

1) Step 1: Estimate the mixed signal's carrier frequency $\hat{f}_{c}$ and bandwidth $\hat{B}$ through spectrum analysis.

2) Step 2: Calculate the SOF around $2 \hat{f}_{c}$ for a cyclic frequency span of $\hat{B}$ with fine resolution.

3) Step 3: Identify the number of signals and estimate the carrier frequencies.

The first step is a simple spectrum analysis and the carrier frequency estimation $\hat{f}_{c}$ is not very accurate. This estimation is only used for the second step to perform high resolution SOF calculation. Although SOF calculation is a computational demanding task with high resolution, we are not obtaining the entire SOF across all cyclic frequency domain. Instead, we are only obtaining SOF at those cyclic frequencies where the SOF features at twice the carrier frequency of each and every individual signal component is expected to exhibit. By doing this, we can significantly reduce the computational complexity.

Fig. 2 shows the SOF of a mixed signal with two BPSK modulated components with $100 \%$ overlap in spectrum. The carrier frequencies are set to $f_{c_{1}}=20000 \mathrm{~Hz}$ and $f_{c_{2}}=20100$ $\mathrm{Hz}$. The symbol rates are set to $f_{b_{1}}=2500 \mathrm{~Hz}$ and $f_{b_{2}}=2400$ $\mathrm{Hz}$. A raised cosine filter with roll-off factor of 1 is used for both transmitted signals. Fig. 3 shows the spectra of both signals. It is clear from Fig. 3 that the second signal's spectrum is entirely contained within the first signal's spectrum with a $100 \%$ overlap. This is the worst case scenario for spectrum overlapping. Here we assume the powers of the two components in the received signal are $3 \mathrm{~dB}$ different from each other.

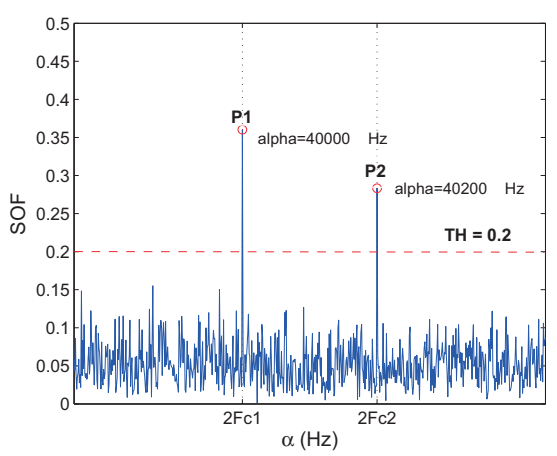

Fig. 2. SOF of two different BPSK signals with $100 \%$ spectrum overlap 


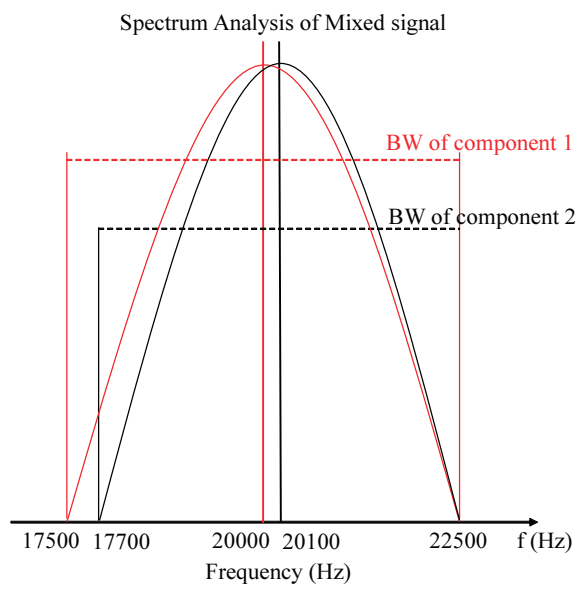

Fig. 3. Spectra of two signals with $100 \%$ spectrum overlap

It is evident from Fig. 2 that the two signal components exhibit two peaks at twice their carrier frequencies as predicted by the cyclostationary analysis theory. It is also clear that the SOF peaks are way above the noise floor, hence we can set a universal threshold to determine the number of signal components present in the target. Here, we simply choose a predetermined threshold of 0.2 and count the number of peaks above this threshold, then estimate the carrier frequency from the cyclic frequencies of such peaks.

\section{Performance of Mixed Signal Detection and CARRIER FREQUENCY ESTIMATION}

In this section, we evaluate the performance of the proposed mixed signal detection and carrier frequency estimation algorithm under various channel conditions, signal to noise ratios, and different signal mixture scenarios.

\section{A. Comparison Between SOF based Algorithm and SCF based Algorithm}

Let's first compare the algorithm proposed in this paper with the previously proposed SCF based detection algorithm in [10]. Fig. 4 and Fig. 5 show the SCF and SOF for the same mixed signal which has two BPSK signal components. The two components have $81.25 \%$ spectrum overlap and the power of the second one is $7 \mathrm{~dB}$ less than the first one. The SNR of mixed-signal is set to be $10 \mathrm{~dB}$ (here we assume SNR as the ratio between total power of received signal and noise power). It is apparent from the figures that the SOF has a better distinction than SCF and therefore is more robust, in addition to the benefit of universal detection threshold.

\section{B. Effects of Different Channel Conditions and Noises}

The most important factor in mixed-signal detection using this method is the difference of received power between different signal components. In a realistic situation, the transmitted power of signals, the distances between transmitters and receiver, and the fading factor in the channels are quite different. As a direct result, at the receiver side, the received

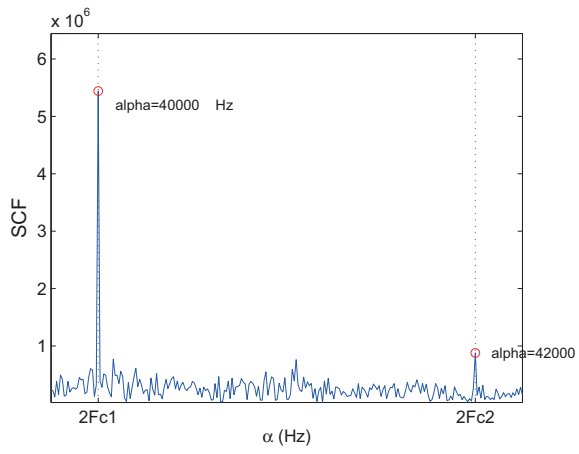

Fig. 4. SCF of two different BPSK signals with $7 \mathrm{~dB}$ received power difference and $81.25 \%$ spectrum overlap

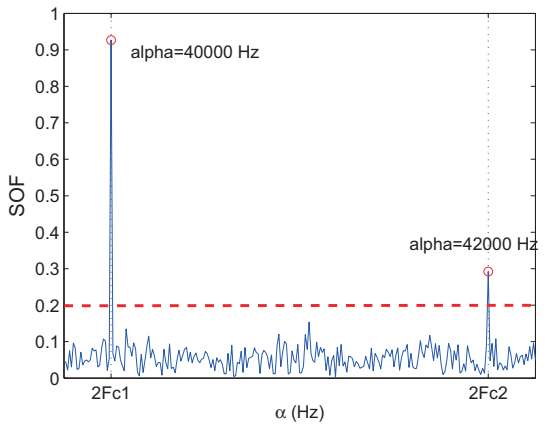

Fig. 5. SOF of two different BPSK signals with $7 \mathrm{~dB}$ received power difference and $81.25 \%$ spectrum overlap

signals' power can be significantly different from others and the weak signal component might be overshadowed by the stronger signal component. Here, we evaluate the performance under different channel conditions.

Fig. 6 shows the SOF of two BPSK signal components with $100 \%$ spectrum overlap. The SNR at receiver is set to be 10 $\mathrm{dB}$. All parameters for these experiments are set to be the same except the relative power of the two components. In Fig. 6(a), the received power of the two components are the same; in Fig. 6(b), the difference of received power between the two components is $5 \mathrm{~dB}$; and in Fig. 6(c), the difference is $10 \mathrm{~dB}$. Clearly, we can find that as the increasing of the received power difference between two components, the detection becomes more difficult. In Fig. 6(c), the detection algorithm fails to detect that there are two signal components.

Hence, let's investigate the detection accuracy as a function of the relative power between the mixed signal components. The dot-curve in Fig. 7 demonstrates the detection accuracy versus the received signal power differences. Both signals are transmitted through independent fading channels and received SNR is $10 \mathrm{~dB}$. If the algorithm correctly detect that there are two signals and accurately estimate their carrier frequency, it is counted as a correct detection, otherwise it is deemed incorrect. As we can see from the figure, when the received power difference is less than $6 \mathrm{~dB}$, our method has excellent accuracy of higher than $90 \%$. As the difference increases 


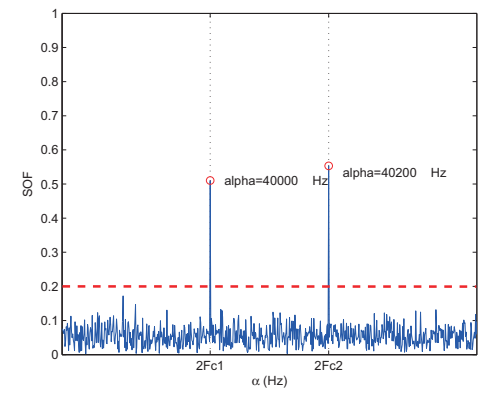

(a) $0 \mathrm{~dB}$ received power difference

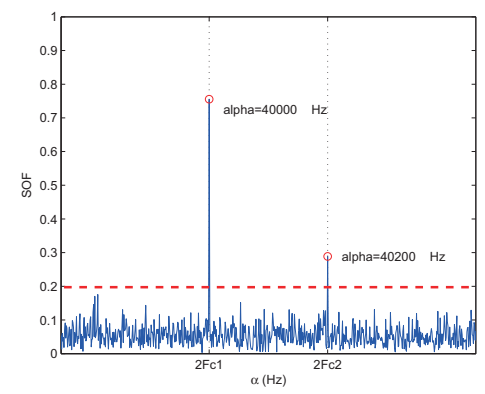

(b) $5 \mathrm{~dB}$ received power difference

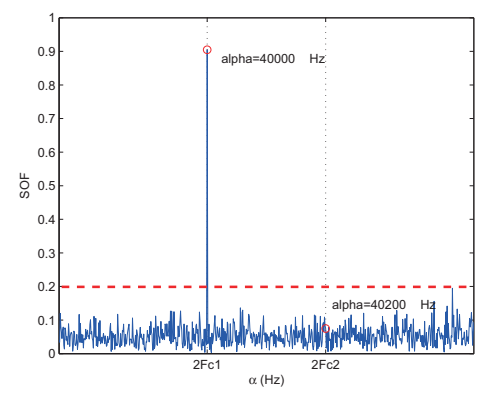

(c) $10 \mathrm{~dB}$ received power difference

Fig. 6. two different BPSK signals with different received power differences and $100 \%$ spectrum overlap

to $10 \mathrm{~dB}$, the detection accuracy gradually falls to around 55\%. The circle-curve in Fig. 7 shows the detection/estimation

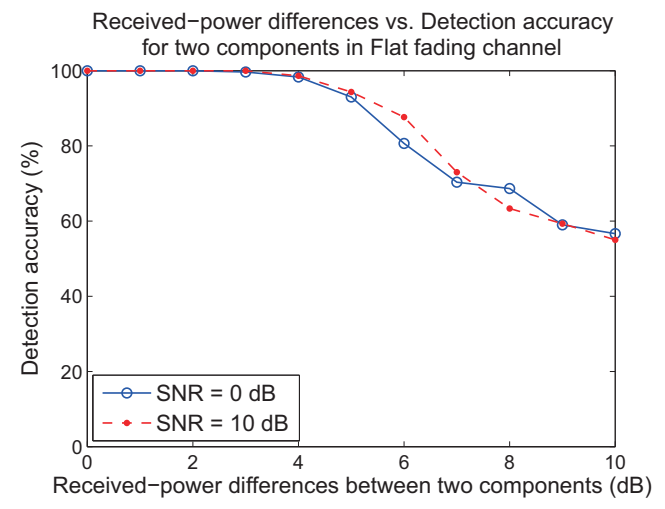

Fig. 7. Received-power differences vs. detection accuracy for two components in Flat fading channel

result when SNR is $0 \mathrm{~dB}$. Compared with the dot-curve, we observe that the SNR of channel has little effect on the performance. This is the direct result of the noise resistance property of cyclostationary analysis, with noise having no spectrum correlation.

\section{Influence of the Signal Length}

As a statistical approach, SOF has excellent performance when the length of signal to be analyzed is long enough. However, it is often desired or required to perform the signal detection and carrier frequency estimation as quickly as possible. Hence, lets evaluate the performance as a function of the signal length.

Fig. 8 demonstrates the results of the detection and estimation accuracy versus the signal length when the mixed signal contains two BPSK components. All of the mixed signals in these experiments are at SNR of OdB. The two signals are overlapped with $100 \%$ spectrum overlap. It is clear from the figure that the detection and estimation accuracy grows as the number of symbols. When the signal power difference between the two mixed components is $0 \mathrm{~dB}$, the accuracy converges to $100 \%$ when the signal length is longer than 4400 symbols. When the signal power difference between the two mixed

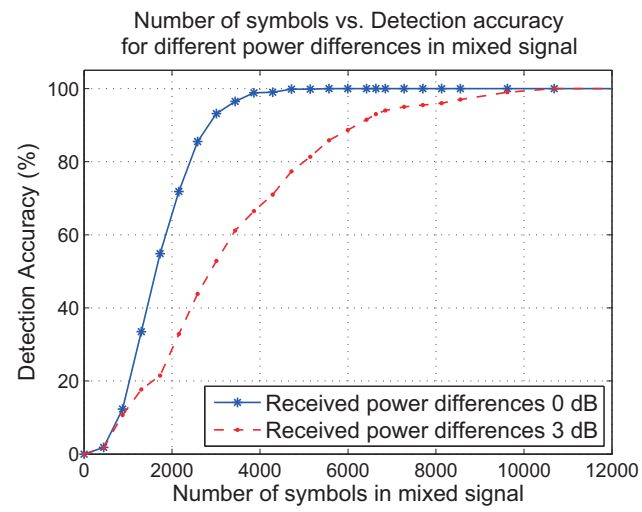

Fig. 8. Detection accuracy vs signal length for 2 components and different signal length in Flat fading channel $(\mathrm{SNR}=0 \mathrm{~dB})$

components is $3 \mathrm{~dB}$, we need 10,000 symbols to reach $100 \%$ accuracy.

\section{Resistance to Different Spectrum Overlap}

Fig. 9 illustrates the SOF of the mixed signal with larger spectrum overlap at $100 \%$ overlap. The received power difference is $7 \mathrm{~dB}$ apart. The received SNR is $20 \mathrm{~dB}$. Compare to Fig. 5 which shows the spectrum overlap at $81.25 \%$ overlap, it is evident that the performances are almost the same. This indicates that the proposed mixed signal detection and carrier frequency estimation algorithm is robust to significant spectrum overlap.

\section{E. Mixed Signal with More Signal Components}

Fig. 10 shows the case of three BPSK modulated signal components in the mixed signal and every two adjacent components have $100 \%$ spectrum overlap. As we can see from the figure, all three components are detected correctly.

However, when the number of mixture components increases, the detection accuracy decreases. Fig. 11 shows that when the largest received-power differences between each two components is larger than $3 \mathrm{~dB}$, the detection accuracy will drop to below $90 \%$. 


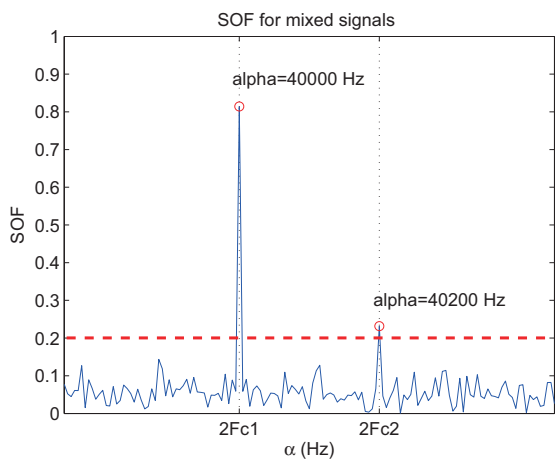

Fig. 9. SOF of two different BPSK signals with $7 \mathrm{~dB}$ received power difference and $100 \%$ spectrum overlap

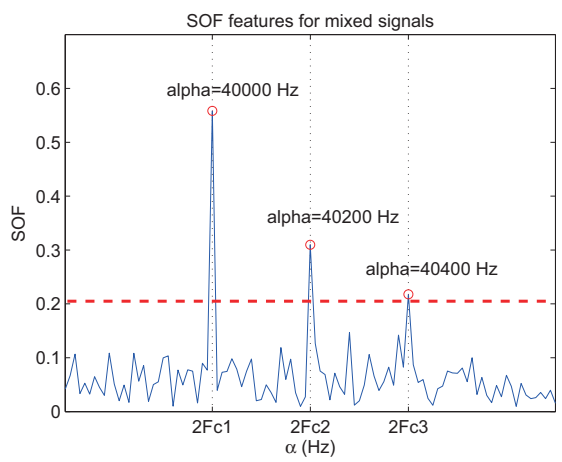

Fig. 10. SOF of three different BPSK signals with $100 \%$ spectrum overlap

\section{CONCLUSION}

In this paper, we develop a robust spectrum coherence function (SOF) based algorithm to detect mixed signals with significant spectrum overlap, and estimate their carrier frequencies accurately. Because of the invariance property of SOF, we can choose a universal threshold for detection. Since additive noise has no spectrum correlation, the proposed SOF based algorithm is resistant to noise. Experiments under different channel conditions and signal mixture scenarios confirm the

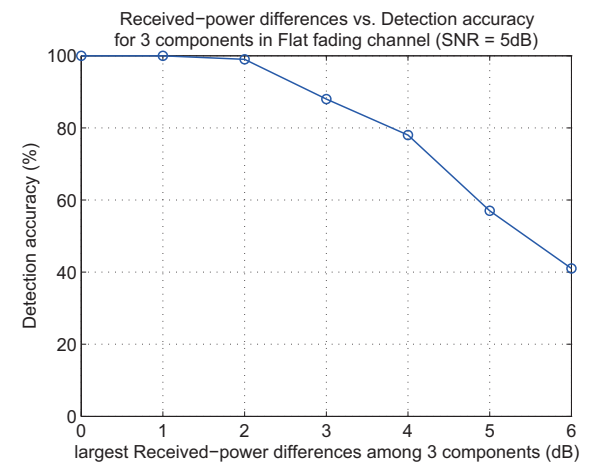

Fig. 11. Largest received-power differences vs. detection accuracy for 3 components in Flat fading channel $(\mathrm{SNR}=5 \mathrm{~dB})$ effectiveness and robustness of the proposed scheme.

\section{ACKNOWLEDGMENT}

This material is based upon work supported by the National Science Foundation under Grant No. 1323240, and the Office of Naval Research. Any opinions, findings, and conclusions or recommendations expressed in this material are those of the author(s) and do not necessarily reflect the views of the funding agencies.

\section{REFERENCES}

[1] V. Chakravarthy, Z. Wu, M. Temple, and F. Garber, "Novel Overlay/Underlay Cognitive Radio Waveforms Using SD-SMSE Framework to Enhance Spectrum Efficiency - Part I: Theoretical Framework and Analysis in AWGN Channel," IEEE Transactions on Communications, vol. 57, no. 12, pp. 3794-3804, December 2009

[2] V. Chakravarthy, Z. Wu and M. Temple, "Novel Overlay/Underlay Cognitive Radio Waveforms Using SD-SMSE Framework to Enhance Spectrum Efficiency - Part II: Analysis in Fading Channel,' IEEE Transactions on Communications, vol. 58, no. 6, pp. 1868-1876, June 2010

[3] Z. Wu and T. C. Yang, "Blind cyclostationary carrier frequency and symbol rate estimation for underwater acoustic communication," Communications (ICC), 2012 IEEE International Conference on, pp. 3482-3486, 2012

[4] Z. Wu and T. C. Yang, "Blind Cyclostationary Carrier Frequency and Symbol Rate Estimation for Underwater Acoustic Communications," IEEE MMTC E-Letter Special Issue on Acoustic and Audio Communication, vol. 7, no. 2, pp. 25-29, February 2012

[5] E. Like, V. Chakravarthy, P. Ratazzi, and Z. Wu, "Signal classification in fading channels using cyclic spectral analysis," EURASIP Journal on Wireless Communications and Networking, pp. 29, September 2009

[6] Z. Wu, T. Yang, Z. Liu, and V. Chakarvarthy, "Modulation detection of underwater acoustic communication signals through cyclostationary analysis," MILITARY COMMUNICATIONS CONFERENCE, 2012-MILCOM 2012, pp. 1-6, 2012

[7] J. Sanderson, X. Li, Z. Liu and Z. Wu, "Hierarchical Blind Modulation Classification for Underwater Acoustic Communication Signal via Cyclostationary and Maximal Likelihood Analysis," Military Communications Conference, MILCOM 2013, pp. 29-34, 2013

[8] R. Zhou, X. Li, T. Yang, Z. Liu and Z. Wu, "Real-time Cyclostationary Analysis for Cognitive Radio via Software Defined Radio," Global Communications Conference (GLOBECOM), 2012 IEEE, pp. 1495-1500, 2012

[9] Y. Qu, X. Li and Z. Wu, "Software-Defined Radio based Automatic Blind Hierarchical Modulation Detector via Second-Order Cyclostationary Analysis and Fourth-Order Cumulant," Military Communications Conference, MILCOM 2013-2013 IEEE, pp. 441-446, 2013

[10] D. Li, Y. Qu, Z. Wu, Z. Liu and Z. Zhang, "Mixed Signal Detection based on Second-order Cyclostationary Features," Military Communications Conference (MILCOM), 2014 IEEE, pp. 682-687, 2014

[11] W. A. Gardner, Cyclostationarity in Communications and Signal Processing, STATISTICAL SIGNAL PROCESSING INC YOUNTVILLE CA, 1994

[12] W. A. Gardner, "The spectral correlation theory of cyclostationary timeseries", Signal Processing, vol. 11, pp. 13-36, July 1986

[13] W. A. Gardner, "Spectral correlation of modulated signals, Part i : Analog modulation," IEEE Transactions on Communications, vol. 35, no. 6 , pp. $584-594,1987$

[14] W. A. Gardner, W. A. Brown, and C.-K. Chen, "Spectral correlation of modulated signals:part ii - digital modulation," IEEE Transactions on Communications, vol. 35, no. 6, pp. 595-601, 1987

[15] W. A. Gardner, "Signal interception: A unifying theoretical framework for feature detection," IEEE Transactions on Communications, vol. 36, no. 8 , pp. $897-906,1988$ 